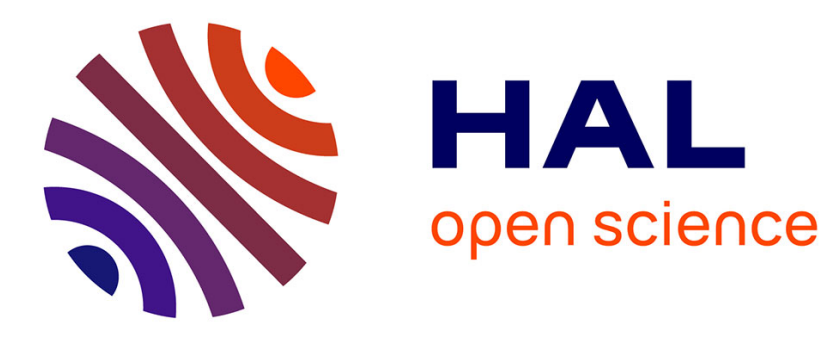

\title{
Les méthodes du bootstrap dans les modèles de régression
}

Emmanuel Flachaire

\section{To cite this version:}

Emmanuel Flachaire. Les méthodes du bootstrap dans les modèles de régression. Economie et Prévision, 2001, 142, pp.183-194. halshs-00175894

\section{HAL Id: halshs-00175894 \\ https://shs.hal.science/halshs-00175894}

Submitted on 1 Oct 2007

HAL is a multi-disciplinary open access archive for the deposit and dissemination of scientific research documents, whether they are published or not. The documents may come from teaching and research institutions in France or abroad, or from public or private research centers.
L'archive ouverte pluridisciplinaire HAL, est destinée au dépôt et à la diffusion de documents scientifiques de niveau recherche, publiés ou non, émanant des établissements d'enseignement et de recherche français ou étrangers, des laboratoires publics ou privés. 


\title{
Les méthodes du bootstrap dans les modèles de régression
}

\author{
Emmanuel Flachaire \\ GREQAM - Université de la Méditerranée
}

Décembre 2000

\begin{abstract}
Résumé
Dans la pratique, la plupart des statistiques de test ont une distribution de probabilité de forme inconnue. Généralement, on utilise leur loi asymptotique comme approximation de la vraie loi. Mais, si l'échantillon dont on dispose n'est pas de taille suffisante cette approximation peut être de mauvaise qualité et les tests basés dessus largement biaisés. Les méthodes du bootstrap permettent d'obtenir une approximation de la vraie loi de la statistique en général plus précise que la loi asymptotique. Elles peuvent également servir à approximer la loi d'une statistique qu'on ne peut pas calculer analytiquement. Dans cet article, nous présentons une méthodologie générale du bootstrap dans le contexte des modèles de régression.
\end{abstract}

\begin{abstract}
In practice, we rarely know the true probability distribution of a test statistic, and we base tests on its asymptotic distribution. If the sample size is not large enough, the asymptotic distribution could be a poor approximation to the true distribution, and consequently tests based on it could be biased. Under mild regularity conditions, bootstrap methods yield an approximation to the distribution of a test statistic that is more accurate than the approximation obtained from first-order asymptotic theory. Moreover they provide a way to substitute computation for mathematical analysis if calculating the asymptotic distribution of an estimator or statistic is difficult. In this paper, we present a general methodology of the bootstrap in regression models.
\end{abstract}

Classification JEL : C1

Mots-clés : bootstrap, modèles de régression

Key-words : bootstrap, regression models

Cet article a été réalisé en partie lors d'un séjour au CORE à l'Université Catholique de Louvain. Je tiens à remercier Russell Davidson pour ses précieux conseils et ses nombreux commentaires ainsi qu'un rapporteur anonyme de la revue. 


\section{Introduction}

La qualité de l'analyse économétrique dépend en tout état de cause de la fiabilité des statistiques de tests employées. Lorsqu'on souhaite faire de l'inférence, c'est-à-dire tester une hypothèse, il est nécessaire de calculer une statistique de test et d'en connaître la loi de probabilité : on peut alors calculer un seuil critique ou une $\boldsymbol{P}$-value et accepter ou non l'hypothèse posée. Malheureusement, la distribution de probabilité d'une statistique de test est la plupart du temps inconnue, à moins de faire des hypothèses fortes et difficilement vérifiables sur le modèle. La théorie asymptotique joue alors un rôle déterminant puisqu'elle permet de relâcher ces hypothèses : les développements asymptotiques au premier ordre déterminent la distribution de probabilité de la statistique lorsque la taille de l'échantillon est infiniment grande, appelée loi asymptotique. En pratique le nombre de données est fini et on utilise la loi asymptotique comme approximation de la vraie loi inconnue. Toutefois, pour que la loi asymptotique soit une bonne image de la vraie loi de la statistique il faut que le nombre de données soit suffisamment important, sinon les tests peuvent être faussés. Une des caractéristiques majeure des méthodes du bootstrap est qu'elles permettent très souvent d'obtenir une meilleure approximation de la vraie loi de la statistique que celle donnée par la loi asymptotique. La fiabilité des tests en est accrue, notamment pour des échantillons dont le nombre d'observations n'est pas très grand. Ces progrès ont des conséquences profondes en sciences car le gain de précision apporté par le bootstrap peut avoir d'importants effets sur les conclusions scientifiques, qui s'appuient sur des évaluations statistiques de la vérité. Les applications du bootstrap sont diverses et variées, en économétrie leur apport principal concerne l'amélioration de l'inférence dans les modèles de régression, comme le souligne le survey de Horowitz (1997).

L'article d'Efron (1979) a été un événement majeur en statistique, d'importants travaux ont par la suite vu le jour de sorte qu'aujourd'hui les applications du bootstrap sont nombreuses. Il existe de nombreux surveys sur le sujet dans la littérature statistique, ainsi que plusieurs ouvrages dont ceux d'Efron et Tibshirani (1993), Shao et Tu (1995), Barbe et Bertail (1995) ou encore Davison et Hinkley (1997). Ce n'est que plus récemment que les économètres se sont intéressés à ces techniques, on peut se référer à l'article de Horowitz (1994) qui applique le bootstrap aux tests de la matrice d'information. L'une des particularités du bootstrap est qu'il en existe de nombreuses mises en oeuvre, qui dépendent étroitement du contexte étudié. L'expérience de sa pratique et les nombreux résultats des simulations sur ses propriétés en échantillon fini montrent qu' en général le bootstrap donne des résultats raisonnables et fiables, mais qu'une utilisation inadéquate peut avoir des conséquences dramatiques. Quelques exemples où le bootstrap ne fonctionne pas ont également été signalés dans la littérature, c'est par exemple le cas pour les $\boldsymbol{U}$-statistiques dégénérées et avec l'estimateur de la borne $\boldsymbol{\theta}$ de la loi uniforme $\boldsymbol{U}(\mathbf{0}, \boldsymbol{\theta})$, voir par exemple Bickel et Freedman (1981). 
Dans le contexte des modèles de régression classiques et pour les statistiques usuelles en économétrie, le bootstrap peut améliorer considérablement la qualité de l'inférence, notamment en petit échantillon. Il faut toutefois rester attentif lors de son utilisation.

L'objectif de cet article est de proposer une méthodologie générale du bootstrap à adopter dans le contexte des modèles de régression, afin d'améliorer la fiabilité des statistiques de test. Nous utilisons la régression linéaire pour simplifier la présentation, les résultats restant valables dans le cadre des régressions non-linéaires, robustes ou non-paramétriques. Sur la base d'un rappel de quelques fondements, nous présentons le principe du bootstrap dans le contexte des modèles de régression, puis ses conditions d'applications, section 2. Nous passons ensuite en revue dans la section 3, ses différentes mises en oeuvre en pratique pour divers cadres d'analyse courants en économétrie : aléas i.i.d. - aléas hétéroscédastiques séries temporelles - modèles sur-identifiés. Dans la section 4, nous exposons l'utilisation du bootstrap pour construire des intervalles de confiance et comparons cette approche à celle des tests d'hypothèses. Finalement, section 5, nous illustrons l'utilisation et les avantages du bootstrap à travers une application empirique.

\section{Le bootstrap}

Avant de présenter le principe général du bootstrap dans un modèle de régression, nous définissons quelques concepts fondamentaux. Ensuite, nous étudions la validité asymptotique et la précision en échantillon fini de ces méthodes.

\section{$2.1 \quad$ Fondements}

En premier lieu, nous donnons les définitions de ce que sont un modèle, un processus générateur de données, une statistique, un pivot et un pivot asymptotique.

On définit tout modèle $\mathcal{M}$ comme un ensemble de processus générateur de données, ou ensemble de PGD. On entend par processus générateur de données, quelque chose qui peut être simulé pour toute taille d'échantillon sur ordinateur, afin de générer un ensemble de données. L'aspect essentiel du PGD est qu'il n'est pas défini à moins que l'on ait suffisamment d'information : les valeurs des paramètres sont connues ainsi que les lois de probabilités. Un exemple simple est le modèle suivant :

$$
y=X \beta+u, \quad E(u \mid X)=0, \quad E\left(u u^{\top} \mid X\right)=\sigma^{2} I
$$

où $\boldsymbol{y}$ est un vecteur de $\boldsymbol{n}$ observations, $\boldsymbol{X}$ une matrice $\boldsymbol{n} \times \boldsymbol{k}$ de régresseurs exogènes, c'est à dire indépendants du terme d'erreur, et $\boldsymbol{u}$ un vecteur aléatoire dont les composantes sont les aléas d'espérance nulle et de covariance proportionnelle à la matrice identité $\boldsymbol{I}$. 
Les paramètres inconnus du modèle sont le $\boldsymbol{k}$-vecteur $\boldsymbol{\beta}$ et le scalaire $\boldsymbol{\sigma}^{2}$. Ce modèle est caractérisé par une infinité de PGD, chaque PGD étant complètement spécifié pour des valeurs de $\boldsymbol{\beta}$ et $\boldsymbol{\sigma}^{2}$ fixés et une loi des aléas définie. Pour un PGD donné $\boldsymbol{\mu}$, il est possible de générer un ensemble de données $\boldsymbol{y}^{\star}$ de la variable dépendante $\boldsymbol{y}$. Une statistique est une fonction des données, c'est par construction une variable aléatoire qui dépend des données $\boldsymbol{y}$ et sa loi dépend du PGD sous-jacent à ces données $\boldsymbol{\mu}$. Une statistique de test est un pivot par rapport à un modèle $\mathcal{M}$ si sa distribution de probabilité est la même pour tout PGD du modèle pour une taille d'échantillon donnée, autrement dit si elle est invariante par rapport aux caractéristiques inconnues du modèle (les paramètres et la loi des aléas dans notre exemple). Une statistique est un pivot asymptotique par rapport au modèle $\mathcal{M}$ si sa loi asymptotique est la même pour tout PGD du modèle.

Nous verrons par la suite que la notion de pivot est essentielle pour comprendre pourquoi le bootstrap fonctionne correctement. L'intérêt est qu'en économétrie, la plupart des statistiques de tests sont des pivots ou des pivots asymptotiques. Considérons par exemple un modèle de régression linéaire avec des régresseurs exogènes. Pour une loi connue des aléas, une statistique qui ne dépend que des régresseurs et des résidus est un pivot. En effet, les résidus sont issus d'une projection orthogonale des aléas sur l'espace engendré par les régresseurs. Dans ce cas, la loi de la statistique est uniquement fonction des régresseurs et de la loi des aléas. Elle est invariante par rapport à tout le reste. Par ailleurs, les statistiques qui suivent (asymptotiquement) les lois de Student, de Fisher, du Chi-deux ou encore la loi Normale centrée réduite, sont des pivots (asymptotiques).

\subsection{Principe}

Les définitions précédentes nous permettent de définir le principe du bootstrap comme suit, Dans les modèles de régression, le principe du bootstrap consiste à spécifier un PGD, nommé PGD bootstrap, en remplaçant les paramètres et distributions de probabilités inconnus dans le modèle, par des estimations empiriques de ces derniers. La distribution de probabilité de la statistique de test sous ce PGD artificiel est appelée loi bootstrap. On calcule un test bootstrap ou une $\boldsymbol{P}$-value bootstrap en utilisant la loi bootstrap pour loi nominale.

L'idée est que si le PGD bootstrap est suffisamment proche du vrai, les données générées par celui-ci seront similaires à celles qui auraient été générées par le vrai PGD. Une $\boldsymbol{P}$-value obtenue avec la loi bootstrap devrait alors être très proche de celle qui aurait été calculée avec la vraie loi de la statistique, de telle sorte que l'inférence est fiable.

On aimerait calculer la loi bootstrap à partir de développements analytiques pour en obtenir une expression exacte. Malheureusement, c'est la plupart du temps impossible. On 
a alors recours à des simulations pour en obtenir une approximation. Plaçons-nous dans le cadre d'un modèle de régression, où un PGD est complètement spécifié pour des valeurs fixes de ses paramètres inconnus $\boldsymbol{\theta}$ et pour une loi de probabilité des aléas donnée $\boldsymbol{F}$. Si on considère par exemple le modèle (1), on aurait $\boldsymbol{\theta}=\left(\boldsymbol{\beta}, \boldsymbol{\sigma}^{2}\right)$. Soit $\boldsymbol{\tau}$ la statistique d'intérêt, dont la loi $G$ est inconnue. La loi bootstrap $G^{\star}$ peut être approximée par simulations à partir de l'algorithme suivant :

1. On calcule des estimateurs $\hat{\boldsymbol{\theta}}$ des paramètres inconnus et $\hat{\boldsymbol{F}}$ de la loi de probabilité des aléas et on calcule une réalisation de la statistique de test $\tau$.

2. On construit le PGD bootstrap $\boldsymbol{\mu}^{\star}$ et on génère des données $\boldsymbol{y}^{\star}$. Sur la base des données simulées, appelées échantillon bootstrap, on calcule une réalisation de la statistique bootstrap $\tau^{\star}$.

3. On répète l'étape précédente $\boldsymbol{B}-\mathbf{1}$ fois afin d'obtenir $\boldsymbol{B}$ réalisations de la statistique bootstrap $\left\{\hat{\tau}_{i}^{\star}\right\}_{i=1 \ldots B}$. La fonction de distribution empirique formée par ces $\boldsymbol{B}$ réalisations est une approximation de la loi bootstrap : $\hat{G}^{\star}(x)=B^{-1} \sum_{i=1}^{B} I\left(\hat{\tau}_{i}^{\star} \leq x\right)$.

$\boldsymbol{I}($.$) est la fonction indicatrice qui prend les valeurs \mathbf{1}$ si son argument est vrai et $\mathbf{0}$ autrement. Suivant l'information que l'on possède sur la loi de probabilité des aléas $\boldsymbol{F}$, on distingue deux méthodes différentes,

- le bootstrap paramétrique : si $\boldsymbol{F}$ appartient à une famille paramétrique connue et de dimension fini $\boldsymbol{F}(\boldsymbol{\delta})$, on utilise $\hat{\boldsymbol{F}}=\boldsymbol{F}(\hat{\boldsymbol{\delta}})$ où $\hat{\boldsymbol{\delta}}$ est un estimateur convergent de $\boldsymbol{\delta}$.

- le bootstrap non-paramétrique : on utilise la fonction de distribution empirique (EDF) des résidus centrés.

Tel que nous l'avons défini, on distingue dans le bootstrap deux types d'approximations. L'approximation théorique : c'est la différence entre la loi bootstrap et la vraie loi, elle provient de l'utilisation d'un PGD bootstrap qui est différent du vrai PGD inconnu. Il va de soi que si la statistique d'intérêt est un pivot par rapport au modèle, la loi bootstrap sera identique à la vraie loi et cette approximation sera inexistante. L'essentiel des travaux théoriques sur le bootstrap porte sur la détermination des taux de convergence de la loi bootstrap vers la vraie loi, lorsque la statistique n'est pas un pivot exact. L' approximation expérimentale apparaît lorsque la loi bootstrap ne peut pas être calculée analytiquement. Cette approximation est complètement indépendante de la première et elle provient du nombre fini de simulations que l'on fait. Utiliser un nombre fini de simulations n'est pas sans conséquences : Hall (1986) montre que les intervalles de confiance bootstrap tendent à être trop longs et Jöckel (1986) montre qu'il y a une perte de puissance des tests. Par ailleurs, Davidson et MacKinnon (2000) montrent que le nombre de simulations bootstrap $\boldsymbol{B}$ doit être choisi tel que $\boldsymbol{\alpha}(\boldsymbol{B}+1)$ soit un entier, où $\boldsymbol{\alpha}$ est le niveau nominal, pour supprimer tout biais éventuel de l'estimation bootstrap d'un seuil critique ou d'une $\boldsymbol{P}$-value. Ils présentent également une procédure de test préliminaire qui permet de minimiser la perte de puissance. 
Si la statistique est un pivot exact et que sa loi bootstrap est approximée par des simulations, les tests bootstrap se confondent avec les tests Monte Carlo : seule l'approximation expérimentale est présente. Hall (1994), Dufour et Kiviet (1998) préfèrent parler de tests Monte Carlo car la littérature sur ce sujet, Dwass (1957), Barnard (1963) et Birnbaum (1974), précède largement celle sur le bootstrap, Efron (1979).

\subsection{Validité}

Pour que le bootstrap soit valide, il faut que la loi bootstrap converge faiblement, en probabilité, vers la vraie loi. Pour comparer deux distributions, la distance de Mallows (1972) est une métrique qui permet de montrer que la distance entre la loi bootstrap et la vraie loi converge vers zéro lorsque le nombre d'observations tend vers l'infini. Cette technique est la plus couramment utilisée dans la littérature statistique pour démontrer la validité asymptotique du bootstrap, voir parmi d'autres Bickel et Freedman (1981) et Freedman (1981). Dans notre optique, nous utilisons principalement le bootstrap dans le but d'obtenir une meilleure approximation de la vraie loi d'une statistique que celle donnée par la loi asymptotique. La connaissance de la loi asymptotique permet de démontrer la validité du bootstrap de manière plus classique : pour une statistique d'intérêt $\boldsymbol{\tau}$ dont on connaît la loi asymptotique $\boldsymbol{G}_{\boldsymbol{a}}$, il suffit de montrer à partir du PGD bootstrap que la loi asymptotique de la statistique bootstrap $\boldsymbol{\tau}^{\star}$ est égale à $\boldsymbol{G}_{\boldsymbol{a s}}$. On peut le faire en utilisant la Loi des Grands Nombres (LGN) et le Théorème Central Limite (TCL) de la même façon qu'on le fait pour déterminer la loi asymptotique de la statistique $\tau$.

\subsection{Précision}

Une fois la convergence établie, il reste à savoir si le bootstrap fonctionne correctement dans la pratique. Nous avons vu que si la statistique est un pivot exact, le bootstrap conduira à des résultats quasiment parfaits car l'approximation théorique est alors inexistante (cf. 2.2). Si la statistique n'est pas un pivot exact, il faut déterminer dans quelle mesure la loi bootstrap est une bonne approximation de la vraie loi de la statistique. Pour cela on étudie les vitesses de convergences et, à cette fin, les techniques de développement d'Edgeworth $(1896,1905)$ ou les inégalités de type Berry-Esseen (qui sont des versions sophistiquées de développements de Taylor) sont utilisées.

Les développements théoriques montrent que si la statistique est un pivot asymptotique, l'erreur de la probabilité de rejet d'un test bootstrap (distorsion du niveau) converge plus rapidement vers zéro que celle d'un test asymptotique. Pour une taille d'échantillon $\boldsymbol{n}$, l'erreur de la probabilité de rejet d'un test basé sur sa loi asymptotique est, en général d'ordre $\boldsymbol{n}^{-\mathbf{1 / 2}}$

pour un test uni-latéral, et d'ordre $\boldsymbol{n}^{-1}$ pour un test bi-latéral. Beran (1988) montre que 
l'utilisation du bootstrap permet de réduire cet ordre d'un facteur $n^{-\mathbf{1 / 2}}$, si la statistique est un pivot asymptotique et si les paramètres et distributions du PGD bootstrap sont des estimateurs convergents des paramètres et distributions inconnus du modèle. Dans certains cas particuliers, cet ordre peut être réduit d'un facteur $\boldsymbol{n}^{-\mathbf{1}}$ voir même plus ; pour une discussion détaillée sur les améliorations asymptotiques du bootstrap, basée sur les développements d'Edgeworth, voir Hall (1992).

Davidson et MacKinnon (1999) montrent qu'une amélioration asymptotique supplémentaire, en général d'ordre $\boldsymbol{n}^{\mathbf{- 1 / 2}}$, peut être obtenue si la statistique qui fait l'objet du bootstrap est asymptotiquement indépendante des composantes aléatoires du PGD bootstrap. Ils montrent qu'une telle indépendance est satisfaite dans le cadre du bootstrap paramétrique si le PGD bootstrap est construit avec les paramètres estimés sous le modèle contraint, c'est-à-dire le modèle qui tient compte de l'hypothèse nulle; et qu'elle peut être également obtenue assez facilement dans le cadre du bootstrap non-paramétrique. Ce résultat justifie de manière théorique l'idée de préférer construire le PGD bootstrap sous l'hypothèse nulle, qui remonte à Beran (1986b) ou encore Beran et Srivastava (1985).

La prise en compte de ces améliorations successives indique que dans de nombreuses circonstances l'amélioration asymptotique des tests bootstrap par rapport aux tests asymptotiques sera d'ordre $\boldsymbol{n}^{-1}$. Ces résultats ne sont qu'asymptotique, aussi ils ne permettent pas d'affirmer qu'en échantillon de petite taille, l'inférence bootstrap sera forcément plus fiable que celle des tests asymptotiques si la statistique est un pivot asymptotique. Néanmoins, si en échantillon fini le comportement de la statistique est proche de celui d'un pivot exact et si cette statistique est quasiment indépendante des composantes aléatoires du PGD bootstrap, il ne fait aucun doute que les tests bootstrap seront beaucoup plus fiables. Dans la littérature, de nombreux travaux étudient à l'aide de simulations le comportement en échantillon fini de différentes statistiques : les résultats expérimentaux montrent que si la statistique est un

pivot asymptotique le bootstrap corrige très souvent la distorsion du niveau des tests basés sur la loi asymptotique, voir parmi d'autres Horowitz (1997).

\section{Mises en ouvre}

La mise en œuvre du bootstrap dépend du contexte considéré. Dans cette section, nous présentons son implémentation dans différents cadres d'analyse couramment rencontrés en économétrie : pour un modèle de régression avec des aléas i.i.d., hétéroscédastiques, nonindépendants et dans les modèles sur-identifiés. Le modèle de régression linéaire est utilisé dans un but pédagogique. Les résultats se généralisent aux régressions non-linéaires, robustes ou non-paramétriques, voir par exemple Huet et Jolivet (1989) et Huet, Jolivet, et Messéan (1990). 


\subsection{Aléas i.i.d.}

Considérons le modèle de régression linéaire suivant,

$$
y_{t}=X_{t} \beta+Z_{t} \gamma+u_{t} \quad u_{t} \sim F\left(0, \sigma^{2}\right)
$$

où $\boldsymbol{y}_{\boldsymbol{t}}$ est l'observation $\boldsymbol{t}$ de la variable dépendante, $\boldsymbol{X}_{\boldsymbol{t}}$ est la $\boldsymbol{t}^{\text {ème }}$ ligne d'une matrice $\boldsymbol{n} \times \boldsymbol{k}$ de variables indépendantes, et $\boldsymbol{Z}_{\boldsymbol{t}}$ est l'observation $\boldsymbol{t}$ d'un vecteur de données indépendantes. Les paramètres inconnues sont le $\boldsymbol{k}$-vecteur $\boldsymbol{\beta}$ et le scalaire $\boldsymbol{\gamma} ; \boldsymbol{u}_{\boldsymbol{t}}$ représente les aléas supposés indépendants du modèle, dont la distribution de probabilité $\boldsymbol{F}$ est d'espérance nulle et de variance $\boldsymbol{\sigma}^{2}$. Considérons par exemple l'hypothèse nulle $\boldsymbol{H}_{0}: \gamma=\gamma_{0}$ et la statistique de student

$$
\tau=\left(\hat{\gamma}-\gamma_{0}\right) / \hat{\boldsymbol{S}}(\hat{\gamma})
$$

où $\hat{\gamma}$ est l'estimateur des moindres carrés ordinaires (OLS) du paramètre $\gamma$ et $\hat{\boldsymbol{S}}(\hat{\gamma})$ son écarttype estimé. Cette statistique suit asymptotiquement la loi Normale centrée réduite : elle est donc un pivot asymptotique. Si le nombre de données n'est pas suffisamment important et si la loi $\boldsymbol{F}$ est inconnue ou si les régresseurs contiennent un retard de la variable dépendante, on peut justifier l'utilisation du bootstrap pour améliorer la fiabilité de l'inférence statistique : la loi bootstrap devrait être une meilleure approximation de la vraie loi de la statistique que la loi asymptotique $N(0,1)$ ou la loi de Student.

La mise en œuvre du bootstrap consiste à construire un PGD bootstrap, qui génère des données qui ressemblent le plus possible aux données réelles. Pour spécifier le PGD bootstrap, il faut trouver des estimations convergentes des paramètres inconnus du modèle et, dans le contexte du bootstrap non-paramétrique, une estimation convergente de la loi des aléas.

- Les paramètres du modèle : on pourrait utiliser n'importe quel estimateur convergent des paramètres. Toutefois, pour bénéficier de l'amélioration asymptotique de Davidson et MacKinnon (1999), il faut que la statistique de test soit asymptotiquement indépendante du processus générateur de données bootstrap (cf. 2.4). Cette hypothèse est respectée si le PGD bootstrap est construit avec les estimateurs des paramètres du modèle qui respecte l'hypothèse nulle, appelé modèle contraint. Cela se justifie par le fait que dans de très nombreux cas, les paramètres estimés sous l'hypothèse nulle $\boldsymbol{H}_{\mathbf{0}}$ sont asymptotiquement indépendants des statistiques qui testent $\boldsymbol{H}_{\mathbf{0}}$. Dans le cadre des tests classiques basés sur une estimation par maximum de vraisemblance, Davidson et MacKinnon (1987) en font une démonstration détaillée, qui peut être étendue aux estimations de type NLS, GMM et autres.

- La loi des aléas : si la loi $\boldsymbol{F}$ est connue à un ensemble de paramètres près, il suffit d'obtenir des estimateurs convergents de ces paramètres, on utilise alors le bootstrap para- 
métrique. Si la loi $\boldsymbol{F}$ est inconnue, on utilise la fonction de distribution empirique (EDF) des résidus centrés, qui est une estimation convergente de la loi des aléas, c'est le bootstrap non-paramétrique. Cette loi $\hat{\boldsymbol{F}}$ est appelée loi de rééchantillonnage car, pour générer les données bootstrap, on effectue des tirages aléatoires dans cette fonction. En général, on ne peut pas utiliser l'EDF des résidus non-centrés : pour que le bootstrap soit valide ils doivent être centrés, de sorte que la loi de rééchantillonnage soit d'espérance nulle. Si ce n'est pas le cas, la loi bootstrap tend vers une loi dont le premier moment n'est pas le même que celui de la loi asymptotique : la distance entre la loi bootstrap et la vraie loi ne converge pas vers zéro (cf. 2.3). Notons que pour un modèle de régression linéaire estimé par moindres carrés ordinaires avec la constante pris comme régresseur, les résidus sont par construction centrés et cette transformation n'est pas nécessaire. D'autre part, une amélioration marginale, portant sur le second moment de la loi de rééchantillonnage peut être obtenue. Elle consiste à restandardiser les résidus, de telle sorte que la variance de la fonction de rééchantillonnage soit si possible un estimateur sans biais de la variance des aléas du modèle. On sait que pour un modèle de régression linéaire avec des aléas i.i.d., la variance des résidus tend à sous estimer la variance des aléas : $\boldsymbol{E}\left(\hat{\boldsymbol{u}}^{\top} \hat{\boldsymbol{u}}\right)=\boldsymbol{\sigma}^{\mathbf{2}}(\boldsymbol{n}-\boldsymbol{k})$, où $\boldsymbol{k}$ est le nombre de régresseurs. On peut corriger ce biais en multipliant les résidus centrés par la racine carrée de $\boldsymbol{n} /(\boldsymbol{n}-\boldsymbol{k})$, on obtient les résidus restandardisés $\hat{\boldsymbol{u}}_{\boldsymbol{t}}^{(\mathbf{1})}$. Une autre solution consiste à utiliser le fait que $\boldsymbol{E}\left(\hat{\boldsymbol{u}}_{\boldsymbol{t}}^{2}\right)=\boldsymbol{\sigma}^{\mathbf{2}}\left(\mathbf{1}-\boldsymbol{h}_{\boldsymbol{t}}\right)$, avec $\boldsymbol{h}_{\boldsymbol{t}}=\boldsymbol{V}_{\boldsymbol{t}}\left(\boldsymbol{V}^{\top} \boldsymbol{V}\right)^{-\mathbf{1}} \boldsymbol{V}_{\boldsymbol{t}}^{\top}$ et $\boldsymbol{V}=[\boldsymbol{X}, \boldsymbol{Z}]$. On utilise alors les résidus restandardisés et centrés :

$$
\hat{u}_{t}^{(2)}=\frac{\hat{u}_{t}}{\left(1-h_{t}\right)^{1 / 2}}-\frac{1}{n} \sum_{s=1}^{n} \frac{\hat{u}_{s}}{\left(1-h_{s}\right)^{1 / 2}}
$$

On divise $\hat{\boldsymbol{u}}_{\boldsymbol{t}}$ par un facteur proportionnel à la racine de sa variance. Les résidus $\hat{\boldsymbol{u}}_{\boldsymbol{t}}$ n'ont pas la même variance, il y a une hétéroscédasticité artificielle. Les résidus restandardisés ont tous la même variance, et sont recentrés. Pour conserver le gain de précision de Davidson et MacKinnon (1999) dans le cadre du bootstrap non-paramétrique, la statistique de test doit être non seulement asymptotiquement indépendante des paramètres du modèle, mais également de la fonction de réechantillonnage $\hat{\boldsymbol{F}}$. Les auteurs montrent que si on utilise la fonction de distribution empirique $\hat{\boldsymbol{F}}$ des résidus, du modèle contraint ou non-contraint, on vérifie cette propriété. Seules des expériences Monte-Carlo montrent que l'utilisation des résidus du modèle contraint plutôt que ceux du modèle non-contraint, apporte un gain de précision très sensible, voir van Giersbergen et Kiviet (1994), Li et Maddala (1993) et Nankervis et Savin (1994).

Le bootstrap classique, ou naïf, non-paramétrique se base donc sur le processus gé- 
nérateur de données bootstrap :

$$
y_{t}^{\star}=X_{t} \tilde{\beta}+Z_{t} \gamma_{0}+u_{t}^{\star} \quad u_{t}^{\star} \sim \operatorname{EDF}\left(\tilde{u}_{t}^{(i)}\right)
$$

où $\tilde{\boldsymbol{\beta}}$ est l'estimateur des paramètres du modèle contraint et $\boldsymbol{u}_{\boldsymbol{t}}^{\star}$ un tirage indépendant avec remise dans les résidus du modèle contraint restandardisés et centrés $\tilde{\boldsymbol{u}}_{\boldsymbol{t}}^{(i)}$, où $\boldsymbol{i}=\mathbf{1}, \mathbf{2}$. La loi bootstrap est la loi de la statistique de test,

$$
\tau^{\star}=\left(\hat{\gamma}^{\star}-\gamma_{0}\right) / \hat{S}\left(\hat{\gamma}^{\star}\right)
$$

où $\hat{\gamma}^{\star}$ est l'estimateur OLS de $\gamma_{0}$ et $\hat{\boldsymbol{S}}\left(\hat{\boldsymbol{\gamma}}^{\star}\right)$ l'estimateur de son écart-type. On peut obtenir par simulation, à l'aide de l'algorithme de la section (2.2), une approximation de la loi bootstrap. Plus loin, dans la section (5), nous verrons une description détaillée de cette mise en oeuvre dans le cadre d'une application.

\subsection{Aléas hétéroscédastiques}

Si les aléas du modèle sont hétéroscédastiques et qu'on ne peut pas obtenir des estimations convergentes des variances des aléas, la méthode du bootstrap classique n'est plus valable. La forme de l'hétéroscédasticité pouvant être fonction des régresseurs, on ne peut pas retirer les résidus indépendamment des régresseurs. Il existe deux méthodes différentes pour contourner cette difficulté.

Le bootstrap par paires proposé par Freedman (1981) consiste à générer un échantillon bootstrap en retirant de façon indépendante et avec remise directement dans le couple régressande/régresseurs, plutôt qu'à partir des résidus. Le principal inconvénient de cette méthode est qu'elle n'est pas valide si les régresseurs contiennent des retards de la variable dépendante, c'est à dire pour la plupart des modèles dynamiques. Par ailleurs, la condition d'indépendance des régresseurs et des aléas dans l'échantillon bootstrap n'est plus respectée puisque les régresseurs sont tirés en même temps que la variable dépendante, les performances numériques sont affectées, voir Horowitz (1997). Flachaire (1999) propose une nouvelle implémentation du bootstrap par paires avec de meilleures propriétés numériques. Toutefois, le problème de la perte d'indépendance des régresseurs et des aléas reste présent.

Le wild bootstrap a été introduit par l'article de Wu (1986) qui est discuté entre autres par Beran (1986a). Par rapport au bootstrap par paires, cette méthode a l'avantage d'être valide pour les modèles dynamiques et de conserver l'hypothèse d'indépendance des régresseurs et des aléas dans l'échantillon bootstrap. Si on considère toujours le modèle (2) 
mais avec des aléas hétéroscédastiques : $\boldsymbol{E}\left(\boldsymbol{u}_{\boldsymbol{t}}^{2} \mid \boldsymbol{X}_{\boldsymbol{t}}, \boldsymbol{Z}_{\boldsymbol{t}}\right)=\boldsymbol{\sigma}_{\boldsymbol{t}}^{2}$, le PGD wild bootstrap est

$$
\boldsymbol{y}_{t}^{\star}=\boldsymbol{X}_{t} \tilde{\boldsymbol{\beta}}+Z_{t} \gamma_{0}+\tilde{\boldsymbol{u}}_{t}^{(2)} \varepsilon_{t}^{\star} \quad \varepsilon_{t}^{\star} \sim \hat{\boldsymbol{F}}(0,1)
$$

où $\tilde{\boldsymbol{\beta}}$ est l'estimateur des paramètres du modèle contraint et $\tilde{\boldsymbol{u}}_{\boldsymbol{t}}^{(\mathbf{2})}$ les résidus du modèle contraint restandardisés et centrés, décrits en (4). Pour que le bootstrap soit valide, il faut

que le rééchantillonnage de $\varepsilon_{t}^{\star}$ s'effectue à partir d'une fonction de distribution quelconque $\hat{\boldsymbol{F}}$ d'espérance nulle et de variance l'unité. La loi Normale centrée réduite est donc appropriée. Mais si on impose également au troisième moment de la loi $\hat{\boldsymbol{F}}$ d'être égal à $\mathbf{1}$, Liu (1988) montre qu'un gain de précision est obtenu dans la cas où la loi des aléas du modèle est asymétrique. Plusieurs constructions de $\hat{\boldsymbol{F}}$ sont possibles, dont la loi bi-atomique $\varepsilon_{t}^{\star}=$ $-(\sqrt{5}-1) / 2$ avec la probabilité $(\sqrt{5}+1) /(2 \sqrt{5})$ et $\varepsilon_{t}^{\star}=(\sqrt{5}+1) / 2$ avec la probabilité $1-(\sqrt{5}+1) /(2 \sqrt{5})$. Davidson et Flachaire (1999) démontrent que la loi bi-atomique symétrique qui prend les valeurs $+\mathbf{1}$ ou $-\mathbf{1}$ avec la même probabilité $\mathbf{1 / 2}$ bénéficie d'une amélioration asymptotique reliée à l'indépendance asymptotique de la statistique qui fait l'objet du bootstrap et du PGD bootstrap. Cette dernière version donne des performances toujours supérieures aux autres choix de $\hat{\boldsymbol{F}}$ proposés dans la littérature, les résultats peuvent être exacts dans certains cas précis.

\subsection{Séries temporelles}

Dans le cadre des séries temporelles stationnaires, si les données sont dépendantes dans le temps, on ne peut pas les retirer de façon indépendante. Le rééchantillonnage doit tenir compte de ce caractère dépendant dans le processus générateur de données.

Le bootstrap récursif est utilisé si le modèle est paramétrique, avec une structure i.i.d. sous-jacente, tel un modèle ARMA. Supposons par exemple qu'une série $\boldsymbol{y}_{\boldsymbol{t}}$ est générée par le modèle $\operatorname{ARMA}(\boldsymbol{p}, \boldsymbol{q})$ suivant,

$$
y_{t}+\alpha_{1} y_{t-1}+\ldots+\alpha_{p} y_{t-p}=u_{t}+\beta_{1} u_{t-1}+\ldots+\beta_{p} u_{t-q}
$$

que l'on peut réécrire $\boldsymbol{A}(\boldsymbol{L}, \boldsymbol{\alpha}) \boldsymbol{y}_{\boldsymbol{t}}=\boldsymbol{B}(\boldsymbol{L}, \boldsymbol{\beta}) \boldsymbol{u}_{\boldsymbol{t}}$, où $\boldsymbol{A}$ et $\boldsymbol{B}$ sont des fonctions connues, $\boldsymbol{L}$ l'opérateur de retards, $\boldsymbol{\alpha}$ et $\boldsymbol{\beta}$ des vecteurs de paramètres, et $\boldsymbol{u}_{\boldsymbol{t}}$ une variable aléatoire indépendante et identiquement distribuée. Un échantillon bootstrap peut être généré récursivement par $\boldsymbol{A}(\boldsymbol{L}, \tilde{\boldsymbol{\alpha}}) \boldsymbol{y}_{t}^{\star}=\boldsymbol{B}(\boldsymbol{L}, \tilde{\boldsymbol{\beta}}) \boldsymbol{u}_{t}^{\star}$, où $\tilde{\boldsymbol{\alpha}}$ et $\tilde{\boldsymbol{\beta}}$ sont des estimateurs convergents de $\boldsymbol{\alpha}$ et $\boldsymbol{\beta}$ du modèle contraint, $\boldsymbol{u}_{t}^{\star}$ est tiré de façon indépendante et avec remise dans une fonction de rééchantillonnage qui estime de façon empirique la loi des aléas, voir la section (3.1). Pour éviter les problèmes au bord, l'initialisation la plus pratique et parfois la seule faisable consiste à conditionner l'échantillon bootstrap par rapport aux premières données observées. 
Par exemple, pour le modèle $\operatorname{AR}(1): \boldsymbol{y}_{\boldsymbol{t}}=\boldsymbol{\rho} \boldsymbol{y}_{\boldsymbol{t}-\mathbf{1}}+\boldsymbol{u}_{\boldsymbol{t}}$, on aurait $\boldsymbol{y}_{1}^{\star}=\tilde{\boldsymbol{\rho}} \boldsymbol{y}_{\mathbf{0}}+\boldsymbol{u}_{\mathbf{1}}^{\star}$, puis $\boldsymbol{y}_{2}^{\star}=\tilde{\boldsymbol{\rho}} \boldsymbol{y}_{1}^{\star}+\boldsymbol{u}_{2}^{\star}$ etc..., l'échantillon bootstrap est conditionné par l'observation $\boldsymbol{y}_{\mathbf{0}}$. Concernant l'étude de la précision du bootstrap, on peut se référer à Freedman (1984) ou Bose (1988).

Le bootstrap par blocs est une généralisation de la méthode du bootstrap pour les séries stationnaires, lorsqu'on n'a aucune information sur la forme de la dépendance des données. Le principe consiste à regrouper les résidus centrés dans des blocs de longueur $\boldsymbol{l}$ afin de capter du mieux possible la dépendance existante. Le rééchantillonnage se fait en tirant de manière indépendante et avec remise ces blocs. Il existe deux manières différentes de construire des blocs. La première, développée par Carlstein (1986), consiste à tirer des blocs disjoints qui sont composés d'observations ne pouvant pas appartenir à un autre bloc, on l'appelle le bootstrap stationnaire. La seconde développée par Künsch (1989), le bootstrap MBB “Moving Block Bootstrap", consiste à tirer des blocs non-disjoints. L'inconvénient principal de ces techniques est qu'elles génèrent des données qui n'ont pas la même structure de dépendance que les données d'origine. La construction de blocs indépendants laisse supposer que les données sont indépendantes après un certain nombre de retards. Dans certaines circonstances les performances numériques peuvent alors être mauvaises, celles-ci étant sensibles au choix de $\boldsymbol{l}$. Ces techniques continuent de faire l'objet d'un grand nombre de travaux dans la littérature.

Concernant les modèles non-stationnaires, il faut prendre de grandes précautions car le bootstrap ne fonctionne pas toujours, voir Basawa, Mallik, McCormick, et Taylor (1989). Dans le cadre d'un test de racine unitaire, le PGD bootstrap doit nécessairement imposer la racine unitaire, c'est à dire respecter l'hypothèse nulle, sinon il n'est pas valide, voir Basawa et al. (1991a, 1991b), Li et Maddala (1996), van Giersbergen (1998) ou encore Bertail (1994) dans la littérature française. Plus que pour bénéficier de l'amélioration asymptotique de Davidson et MacKinnon (1999), cette condition est indispensable pour la validité asymptotique du test bootstrap de racine unitaire.

Pour plus de détails concernant le bootstrap dans les modèles dynamiques, on peut se référer au survey de Li et Maddala (1996) et à la thèse de van Giersbergen (1998), qui considèrent également les modèles de cointégration.

\subsection{Modèle sur-identifié et autres cas}

L'estimation par variables instrumentales repose entre autres sur l'hypothèse d'absence de corrélation entre les instruments et les aléas : $\boldsymbol{E}\left(\boldsymbol{W}^{\top} \boldsymbol{u}\right)=\mathbf{0}$, où $\boldsymbol{W}$ est la matrice $\boldsymbol{n} \times \boldsymbol{l}$ des instruments et $\boldsymbol{u}$ le vecteur des aléas. Dans le cadre du bootstrap classique, cette hypothèse n'est pas respectée : $\boldsymbol{E}\left(\boldsymbol{W}^{\top} \boldsymbol{u}^{\star}\right)=\boldsymbol{W}^{\top} \tilde{\boldsymbol{u}}=\boldsymbol{K}$, puisque $\boldsymbol{K}$ est nul seulement si le nombre d'instruments est égal au nombre de régresseurs $\boldsymbol{l}=\boldsymbol{k}$. Si le modèle est sur-identifié, il faut 
donc modifier l'implémentation du bootstrap de manière à ce que le PGD bootstrap respecte cette hypothèse. Freedman (1984) est le premier à traiter ce problème dans le cadre d'une estimation 2SLS et Freedman et Peters (1984a, 1984b) proposent une illustration de ces techniques. Dans le cadre d'une estimation par variables instrumentales (IV), la solution la plus simple consiste à projeter orthogonalement $\tilde{\boldsymbol{u}}$ sur l'espace engendré par les instruments et retenir le complément de cette projection : $\boldsymbol{M}_{\boldsymbol{W}} \tilde{\boldsymbol{u}}$, où $\boldsymbol{M}_{\boldsymbol{W}}=\boldsymbol{I}-\boldsymbol{W}\left(\boldsymbol{W}^{\top} \boldsymbol{W}\right)^{-1} \boldsymbol{W}^{\top}$. Les aléas du PGD bootstrap $\boldsymbol{u}^{\star}$ sont retirés dans les résidus restandardisés et centrés $\boldsymbol{M}_{\boldsymbol{W}} \tilde{\boldsymbol{u}}$.

De manière générale, presque tous les types de modèles économétriques ont une version appropriée du bootstrap : les modèles de régression avec des aléas hétéroscédastiques et/ou autocorrélés, les modèles de régression sans lien apparent, les modèles avec des retards de la variable dépendante, les modèles à données de panel, les modèles à équations simultanées, les logit, probit, tobit et autres modèles à variable dépendante limitée, les estimateurs robustes, les modèles de survie, les estimations semi-paramétriques voire non-paramétriques, les tests de racine unitaire et les modèles de cointégration. Pour plus d'informations, les articles de Jeong et Maddala (1993) et Vinod (1993) font le point sur l'ensemble de ces applications.

\section{Les intervalles de confiance}

Il existe une relation duale entre les intervalles de confiance et les tests d'hypothèses : un intervalle de confiance avec un niveau de confiance $\mathbf{1}-\boldsymbol{\alpha}$ contient l'ensemble des valeurs des paramètres qui ne rejettent pas l'hypothèse nulle au niveau nominal $\boldsymbol{\alpha}$. Dans cette section, nous présentons la construction des intervalles de confiance bootstrap, puis nous montrons que la dualité avec les tests d'hypothèse n'est plus vérifiée lorsqu'on utilise le bootstrap.

\subsection{Le bootstrap}

Par construction, un intervalle de confiance bi-latéral est un intervalle dans lequel la vraie valeur $\beta_{0}$ du paramètre d'intérêt se trouve $100(1-2 \alpha) \%$ de fois. Dans le contexte des modèles de régression, on calcule un estimateur $\hat{\boldsymbol{\beta}}$ du paramètre d'intérêt, un estimateur $\hat{\boldsymbol{S}}(\hat{\boldsymbol{\beta}})$ de son écart-type et une valeur critique $\boldsymbol{c}_{\boldsymbol{\alpha}}$ issue en général de la loi de Student, on construit habituellement l'intervalle de confiance comme suit :

$$
\left[\hat{\boldsymbol{\beta}}-c_{\alpha} \hat{S}(\hat{\boldsymbol{\beta}}) \quad ; \quad \hat{\boldsymbol{\beta}}+c_{\alpha} \hat{S}(\hat{\boldsymbol{\beta}})\right]
$$

Cet intervalle de confiance est exact dans le cas particulier où le modèle de régression est

linéaire avec des régresseurs exogènes et des aléas Normaux : la quantité $\left(\hat{\boldsymbol{\beta}}-\boldsymbol{\beta}_{0}\right) / \hat{\boldsymbol{S}}(\hat{\boldsymbol{\beta}})$ suit alors la loi de Student. Dans tous les autres cas, c'est un intervalle de confiance approximé.

On utilise le bootstrap pour améliorer la qualité de l'approximation. La méthode du 
bootstrap la plus simple et la plus performante est la méthode percentile-t. Son principe consiste à calculer les seuils critiques, non pas à partir de la loi de Student, mais à partir de la loi bootstrap de la quantité $\boldsymbol{\zeta}^{\star}=\left(\hat{\boldsymbol{\beta}}^{\star}-\hat{\boldsymbol{\beta}}\right) / \hat{\boldsymbol{S}}\left(\hat{\boldsymbol{\beta}}^{\star}\right)$, qui est un pivot asymptotique. On construit alors l'intervalle de confiance :

$$
\left[\hat{\boldsymbol{\beta}}-c_{1-\alpha}^{\star} \hat{S}(\hat{\boldsymbol{\beta}}) \quad ; \quad \hat{\beta}+c_{\alpha}^{\star} \hat{S}(\hat{\boldsymbol{\beta}})\right]
$$

où $c_{1-\alpha}^{\star}$ et $c_{\alpha}^{\star}$ sont respectivement les percentiles $\mathbf{1 0 0}(1-\alpha)$ et $\mathbf{1 0 0} \boldsymbol{\alpha}$ de la loi bootstrap. Cet intervalle tient compte d'une possible asymétrie de la distribution de probabilité.

Une méthode alternative à la méthode percentile- $\boldsymbol{t}$ est celle proposée par Efron (1987), appelée Bias Corrected accelerated confidence intervals, ou $\boldsymbol{B} \boldsymbol{C}_{\boldsymbol{a}}$. Cependant, sa mise en oeuvre est plus complexe que la méthode percentile- $\boldsymbol{t}$ sans qu'elle soit pour autant plus performante. Elle peut être utile lorsqu'il n'est pas possible d'avoir une standardisation adéquate de la quantité $\zeta^{\star}$, pour un exposé détaillé et la comparaison de ces différentes méthodes voir Hall (1992).

\subsection{Relation duale}

La construction des intervalles de confiance bootstrap avec la méthode percentile- $\boldsymbol{t}$ est très proche de l'utilisation du bootstrap dans le cadre des tests d'hypothèses. Toutefois, nous allons montrer que la relation duale entre les deux approches n'est en général plus vérifiée lorsqu'on utilise le bootstrap. Considérons le modèle de régression non-linéaire :

$$
y=x(\alpha, \beta)+u \quad u \sim F\left(0, \sigma^{2}\right)
$$

où $\boldsymbol{y}$ est la variable dépendante et $\boldsymbol{x}(\boldsymbol{\alpha}, \boldsymbol{\beta})$ une fonction de régression qui détermine la valeur moyenne de $\boldsymbol{y}$ conditionnellement à $\boldsymbol{\alpha}, \boldsymbol{\beta}$ et un ensemble de régresseurs exogènes $\boldsymbol{Z} ; \boldsymbol{u}$ est un vecteur à $\boldsymbol{n}$ composantes supposées i.i.d., suivant une distribution $\boldsymbol{F}$ inconnue centrée et de variance finie $\boldsymbol{\sigma}^{2}$.

- L'approche en terme des tests d'hypothèse : soit l'hypothèse nulle $\boldsymbol{H}_{\mathbf{0}}: \boldsymbol{\beta}=\boldsymbol{\beta}_{\mathbf{0}}$, on estime le modèle (11) et on calcule la statistique de test $\boldsymbol{\tau}=\left(\hat{\boldsymbol{\beta}}-\boldsymbol{\beta}_{0}\right) / \hat{\boldsymbol{S}}(\hat{\boldsymbol{\beta}})$, où $\hat{\boldsymbol{\beta}}$ est l'estimateur du paramètre $\boldsymbol{\beta}$ et $\hat{\boldsymbol{S}}(\hat{\boldsymbol{\beta}})$ l'estimateur de son écart-type. Le PGD bootstrap est le suivant :

$$
y^{\star}=x\left(\tilde{\alpha}, \beta_{0}\right)+u^{\star} \quad u^{\star} \sim E D F\left(\tilde{u}_{t}^{(2)}\right)
$$

où $\tilde{\boldsymbol{\alpha}}$ est l'estimateur du modèle contraint et $\tilde{\boldsymbol{u}}_{t}^{(2)}$ les résidus restandardisés et centrés du 
modèle contraint, voir (4). On approxime par simulations la loi de la statistique bootstrap

$$
\tau^{\star}=\left(\hat{\boldsymbol{\beta}}_{0}^{\star}-\boldsymbol{\beta}_{0}\right) / \hat{\boldsymbol{S}}\left(\hat{\boldsymbol{\beta}}_{0}^{\star}\right)
$$

où $\hat{\boldsymbol{\beta}}_{\mathbf{0}}^{\star}$ est l'estimateur bootstrap de $\boldsymbol{\beta}_{\mathbf{0}}$ et $\boldsymbol{S}\left(\hat{\boldsymbol{\beta}}_{\mathbf{0}}^{\star}\right)$ un estimateur de son écart-type.

- L'approche en terme des intervalles de confiance : on estime le modèle (11) et on construit le PGD bootstrap comme suit,

$$
y^{\star}=x(\hat{\alpha}, \hat{\beta})+u^{\star} \quad u^{\star} \sim E D F\left(\hat{\boldsymbol{u}}_{t}^{(2)}\right)
$$

où $\hat{\boldsymbol{\alpha}}$ et $\hat{\boldsymbol{\beta}}$ sont les estimateurs du modèle non-contraint (11) et $\hat{\boldsymbol{u}}_{t}^{(2)}$ les résidus restandardisés et centrés du modèle non-contraint, voir (4). On approxime par simulations la loi de la quantité bootstrap

$$
\zeta^{\star}=\left(\hat{\boldsymbol{\beta}}^{\star}-\hat{\boldsymbol{\beta}}\right) / \hat{\boldsymbol{S}}\left(\hat{\boldsymbol{\beta}}^{\star}\right)
$$

où $\hat{\boldsymbol{\beta}}^{\star}$ est l'estimateur bootstrap de $\hat{\boldsymbol{\beta}}$ et $\boldsymbol{S}\left(\hat{\boldsymbol{\beta}}^{\star}\right)$ un estimateur de son écart-type.

Dans l'approche en terme des intervalles de confiance, la statistique d'intérêt $\zeta^{\star}$ n'est pas asymptotiquement indépendante du PGD bootstrap car ce dernier ne respecte pas l'hypothèse nulle, on peut donc s'attendre à ne pas bénéficier du gain de précision de Davidson et MacKinnon (1999) qui est garantie dans l'approche en terme des tests d'hypothèse, voir les sections (2.4) et (3.1). Cela n'a pas d'impact dans le cadre d'un modèle de régression linéaire statique lorsque la statistique de test ne dépend que des régresseurs et des résidus mais, à partir du moment où le modèle est dynamique et/ou non-linéaire, la dualité entre les deux approches n'est plus vérifiée, les résultats expérimentaux de van Giersbergen (1998) le confirment.

\section{Application}

Dans cette section, nous illustrons l'utilisation du bootstrap dans la pratique, à travers un exemple numérique. Le modèle utilisé concerne la demande d'électricité tel que le considère Berndt(1990, exercice 3, p. 339). Les données qu'il utilise décrivent la consommation résidentielle d'électricité de 42 villes de Grande-Bretagne à une date donnée, elles sont disponibles dans la disquette fournie avec son manuel. Il s'agit donc d'un modèle statique avec des données individuelles. Le modèle de régression sur lequel on travaille est le suivant,

$$
K W H_{t}=\beta_{0}+\beta_{1} I N C_{t}+\beta_{2}\left(1 / M C 6_{t}\right)+\beta_{3} G A S 6_{t}+\beta_{4} C A P_{t}+u_{t}
$$


où $\boldsymbol{K} \boldsymbol{W} \boldsymbol{H}$ est la consommation domestique d'électricité par client dans chaque ville, $\boldsymbol{I} \boldsymbol{N} \boldsymbol{C}$ le revenu moyen des consommateurs, $\boldsymbol{M C 6}$ le coût marginal de l'électricité, $\boldsymbol{G A S 6}$ le coût marginal du gaz, et $\boldsymbol{C A P}$ la capacité de production d'électricité. L'estimation de cette régression linéaire peut être effectuée par la méthode des moindres carrés ordinaires si on suppose que les aléas $\boldsymbol{u}_{\boldsymbol{t}}$ sont indépendants et identiquement distribués. On réécrit le modèle (16) de manière simplifiée,

$$
Y_{t}=X_{t} \beta+u_{t}
$$

où $Y_{t}=K W H_{t}, X_{t}=\left(1, I N C_{t}, 1 / M C 6_{t}, G A S 6_{t}, C A P_{t}\right)$ est une ligne de la matrice $\boldsymbol{n} \times \mathbf{5}$ qui regroupe l'ensemble des régresseurs, et $\boldsymbol{\beta}=\left(\boldsymbol{\beta}_{0}, \boldsymbol{\beta}_{1}, \boldsymbol{\beta}_{2}, \boldsymbol{\beta}_{3}, \boldsymbol{\beta}_{4}\right)$. Pour mettre en valeur les avantages du bootstrap, nous choisissons dans un premier temps d'utiliser un échantillon de plus petite taille, où seules les 31 premières données sont utilisées.

Berndt (1990, p. 340) considère que la variance de la consommation domestique d'électricité pourrait être une fonction du nombre de consommateurs par villes : $\boldsymbol{\sigma}^{2} / \boldsymbol{C} \boldsymbol{S} \boldsymbol{T}_{\boldsymbol{t}}$. Une manière de tester cette hypothèse d'hétéroscédasticité des aléas, proposée par White (1980), consiste à régresser les résidus du modèle de départ élevés au carré sur la constante et les variables susceptibles d'expliquer l'hétéroscédasticité, puis tester à l'aide d'une statistique de Fisher la nullité des paramètres associés aux explicatives autres que la constante. Dans notre exemple le test se construit en estimant le modèle (17) par OLS, puis en régressant les résidus au carré sur la constante et la variable influente : $\hat{\boldsymbol{u}}_{t}^{2}=\gamma_{0}+\gamma_{1}\left(1 / C \boldsymbol{U} \boldsymbol{S} \boldsymbol{T}_{t}\right)+\varepsilon_{t}$, où $\varepsilon_{t}$ est un bruit blanc. Le student associé à $\gamma_{1}$ teste notre hypothèse et on peut calculer une $\boldsymbol{P}$-value de cette statistique élevée au carrée et basée sur la loi de Fisher. Le calcul de la $\boldsymbol{P}$-value bootstrap consiste en les étapes qui suivent :

1. Estimer par OLS le modèle (17) de manière à obtenir les paramètres estimés $\hat{\boldsymbol{\beta}}$ et les résidus $\hat{\boldsymbol{u}}$, puis calculer les résidus restandardisés et centrés à partir de la formule (4). Estimer par OLS les résidus au carré sur la constante et $\mathbf{1} / \boldsymbol{C U} \boldsymbol{S} \boldsymbol{T}$ de manière à obtenir une réalisation de la statistique $\hat{\boldsymbol{\tau}}$ qui teste $\boldsymbol{H}_{\mathbf{0}}: \gamma_{\mathbf{1}}=\mathbf{0}$.

2. Générer un échantillon bootstrap à partir du PGD bootstrap $\boldsymbol{Y}_{t}^{\star}=\boldsymbol{X}_{\boldsymbol{t}} \hat{\boldsymbol{\beta}}+\boldsymbol{u}_{t}^{\star}$ où $\boldsymbol{u}_{t}^{\star}$ est un tirage aléatoire dans les résidus restandardisés et centrés. À partir de cet échantillon calculer une nouvelle réalisation de la statistique $\hat{\tau}^{\star}$ comme décrit précédemment.

3. Répéter l'étape précédente un grand nombre de fois de manière à obtenir $\boldsymbol{B}$ statistiques bootstrap $\hat{\tau}_{j}^{\star}, j=1, \ldots, B$. Le nombre de fois où $\hat{\tau}_{j}^{\star}$ est plus grand que $\hat{\boldsymbol{\tau}}$, divisé par $\boldsymbol{B}$, est la $\boldsymbol{P}$-value bootstrap.

La $\boldsymbol{P}$-value obtenue avec la loi de Fisher est $\boldsymbol{P}_{\boldsymbol{f}}=\mathbf{0 . 0 7 2}$ et avec la loi bootstrap $\boldsymbol{P}_{\boldsymbol{b}}=\mathbf{0 . 0 3 6}$, pour $\boldsymbol{B}=9999$. Pour un seuil de confiance à $5 \%$ les conclusions sont opposées : avec la loi asymptotique on ne peut pas rejeter l'hypothèse nulle alors qu'avec la loi bootstrap on 
la rejette. Si on refait l'expérience avec toutes les données $\boldsymbol{n}=\mathbf{4 2}$ on trouve $\boldsymbol{P}_{\boldsymbol{f}}=\mathbf{0 . 0 0 0 4}$ et $\boldsymbol{P}_{\boldsymbol{b}}=\mathbf{0 . 0 0 2 3}$ et dans les deux cas on rejette l'hypothèse nulle. Comme la théorie le suggère, il semble plus efficace d'utiliser la $\boldsymbol{P}$-value bootstrap qui tend plus rapidement vers les bonnes conclusions statistiques.

\section{Références}

Barbe, P. et P. Bertail (1995). The Weighted Bootstrap. New York : Springer-Verlag.

Barnard, G. A. (1963). "Discussion on M. S. Bartlett, the spectral analysis of point processes". Journal of the Royal Statistical Society B, 25, 294.

Basawa, I. V., A. K. Mallik, W. P. McCormick, J. H. Reeves, et R. L. Taylor (1991a). "Bootstrapping test of significance and sequential bootstrap estimation for unstable first order autoregressive processes". Communications in Statistics-Theory and Methods, 20, 1015-1026.

Basawa, I. V., A. K. Mallik, W. P. McCormick, J. H. Reeves, et R. L. Taylor (1991b). "Bootstrapping unstable first order autoregressive processes". Annals of Statistics, 19, 1098-1101.

Basawa, I. V., A. K. Mallik, W. P. McCormick, et R. L. Taylor (1989). "Bootstrapping explosive autoregressive processes". Annals of Statistics, 17, 1479-1486.

Beran, R. (1986a). Discussion of "Jackknife bootstrap and other resampling methods in regression analysis" by C.F.J. Wu. Annals of Statistics, 14, 1295-1298.

Beran, R. (1986b). "Simulating power functions". Annals of Statistics, 14, 151-173.

Beran, R. (1988). "Prepivoting test statistics : a bootstrap view of asymptotic refinements". Journal of the American Statistical Association , 83, 687-697.

Beran, R. et Srivastava (1985). "Bootstrap tests and confidence regions for functions of a covariance matrix". Annals of Statistics, 13, 95-115.

Berndt, E. R. (1990). The Practice of Econometrics : Classic and Contemporary. Addison Wesley.

Bertail, P. (1994). "Un test bootstrap dans un modèle AR(1)". Annales d'Économie et de Statistique, 36, 57-79.

Bickel, P. J. et D. A. Freedman (1981). "Some asymptotic theory for the bootstrap". Annals of Statistics, 9, 1196-1217.

Birnbaum, Z. W. (1974). "Computers and unconventionnal test-statistics". In Reliability and Biometry, pp. 441-458. F. Proschan and R. J. Serfling (eds). Philadelphia : SIAM. 
Bose, A. (1988). "Edgeworth correction by bootstrap in autoregressions". Annals of Statistics, 16, 1709-1722.

Carlstein, E. (1986). "The use of subseries methods for estimating the variance of a general statistic from a stationary time series". Annals of Statistics, 14, 1171-1179.

Davidson, R. et E. Flachaire (1999). "The wild bootstrap, tamed at last". mimeo GREQAM 99A32.

Davidson, R. et J. G. MacKinnon (1987). "Implicit altenatives and the local power of test statistics". Econometrica, 55, 1305-29.

Davidson, R. et J. G. MacKinnon (1999). "The size distortion of bootstrap tests". Econometric Theory, 15, 361-376.

Davidson, R. et J. G. MacKinnon (2000). "Bootstrap tests : How many bootstraps ?". Econometric Reviews, 19, 55-68.

Davison, A. C. et D. V. Hinkley (1997). Bootstrap Methods and their Application. Cambridge : Cambridge University Press.

Dufour, J. M. et J. F. Kiviet (1998). "Exact inference methods for first-order autoregressive distributed lag models". Econometrica, 66, 79-104.

Dwass, M. (1957). "Modified randomization tests for non-parametric hypotheses". Annals of Mathematical Statistics, 28, 181-187.

Edgeworth, F. Y. (1896). "The asymmetrical probability curve". Philos. Mag., 5th Ser., 41, 90-99.

Edgeworth, F. Y. (1905). "The law of error". Proc. Cambridge Philos. Soc., 20, 36-65.

Efron, B. (1979). "Bootstrap methods : another look at the jacknife". Annals of Statistics, 7, $1-26$.

Efron, B. (1987). "Better bootstrap confidence intervals". Journal of the American Statistical Association, 82, 171-200.

Efron, B. et R. Tibshirani (1993). An Introduction to the Bootstrap. New York : Chapman \& Hall.

Flachaire, E. (1999). "A better way to bootstrap pairs". Economics Letters, 64, 257-262.

Freedman, D. A. (1981). "Bootstrapping regression models". Annals of Statistics, 9, 12181228.

Freedman, D. A. (1984). "On bootstrapping two-stage least-squares estimates in stationary linear models". Annals of Statistics, 12, 827-842.

Freedman, D. A. et S. C. Peters (1984a). "Bootstrapping a regression equation : some empirical results". Journal of the American Statistical Association, 79, 97-106. 
Freedman, D. A. et S. C. Peters (1984b). "Bootstrapping an econometric model : some empirical results". Journal of Business \& Economics Statistics , 2, 150-158.

Hall, P. (1986). "On the number of bootstrap simulations required to construct a confidence interval". Annals of Statistics, 14, 1453-1462.

Hall, P. (1992). The Bootstrap and Edgeworth Expansion . Springer Series in Statistics. New York : Springer Verlag.

Hall, P. (1994). "Methodology and theory for the bootstrap". In Handbook of Econometrics, Volume 4, pp. 2341-2379. R. F. Engle and D. L. McFadden (eds), Amsterdam : Elsevier.

Horowitz, J. L. (1994). "Bootstrap-based critical values for the information matrix test". Journal of Econometrics, 61, 395-411.

Horowitz, J. L. (1997). "Bootstrap methods in econometrics : theory and numerical performance". In Advances in Economics and Econometrics : Theory and Application, Volume 3, pp. 188-222. David M. Kreps and Kenneth F. Wallis (eds), Cambridge, Cambridge University Press.

Huet, S. et E. Jolivet (1989). "Bootstrap and edgeworth expansion : the non-linear regression as an example". Rapport technique, Département de Biométrie, INRA.

Huet, S., E. Jolivet, et A. Messéan (1990). "Some simulations results about confidence intervals and bootstrap methods in nonlinear regressions". Statistics, 21, 369-432.

Jeong, J. et G. S. Maddala (1993). "A perspective on application of bootstrap methods in econometrics". In Handbook of Statistics, Volume 11, pp. 573-610. North Holland Publishing Co.

Jöckel, K. H. (1986). "Finite sample properties and asymptotic efficiency of Monte Carlo tests". Annals of Statistics, 14, 336-347.

Künsch, H. R. (1989). "The jacknife and the bootstrap for general stationary observations". Annals of Statistics, 17, 1217-1241.

Li, H. et G. S. Maddala (1993, dec). "Bootstrapping cointegrating regressions". Paper presented at the Fourth Meeting of the European Conference Series in Quantitative Economics and Econometrics.

Li, H. et G. S. Maddala (1996). "Bootstrapping time series models". Econometric Reviews, 15, 115-158.

Liu, R. Y. (1988). "Bootstrap procedure under some non-i.i.d. models". Annals of Statistics, 16, 1696-1708.

Mallows, C. L. (1972). "A note on asymptotic joint normality". The Annals of Mathematical Statistics, 43, 508-515. 
Nankervis, J. C. et N. E. Savin (1994). "The level and power of the bootstrap- $\boldsymbol{t}$ test in the AR(1) model with trend". Manuscript, Department of Economics, University of Surrey and University of Iowa.

Shao, J. et D. Tu (1995). The Jackknife and Bootstrap. New York : Springer-Verlag.

van Giersbergen, N. P. A. (1998). Bootstrapping Dynamic Econometric Models . Ph. D. thesis, University of Amsterdam. Book no. 184 of the Tinbergen Institute Research Series.

van Giersbergen, N. P. A. et J. F. Kiviet (1994). "How to implement bootstrap hypothesis testing in static and dynamic regression model". Discussion paper TI 94-130, Amsterdam : Tinbergen Institute. Paper presented at ESEM'94 and $\boldsymbol{E C}^{\mathbf{2}}$ '93.

Vinod, H. D. (1993). Bootstrap methods : applications in econometrics. In C. R. Rao G.S. Maddala et H.D. Vinod (Eds.), Handbook of Statistics, Volume 11, pp. 629-661. Amsterdam : North Holland.

White, H. (1980). "A heteroskedasticity-consistent covariance matrix estimator and a direct test for heteroskedasticity". Econometrica, 48, 817-838.

Wu, C. F. J. (1986). "Jackknife bootstrap and other resampling methods in regression analysis". Annals of Statistics, 14, 1261-1295. 